\title{
NISTIR 7345
}

\section{Screening Candidates for $30 \mathrm{~nm}$ Spheres}

Michelle K. Donnelly

Jiann C. Yang 
NISTIR 7345

\section{Screening Candidates for $30 \mathrm{~nm}$ Spheres}

Michelle K. Donnelly

Jiann C. Yang

Building and Fire Research Laboratory

August 2006

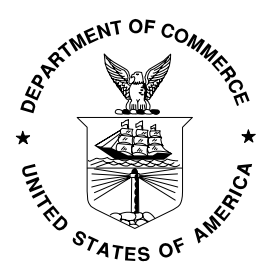

U.S. Department of Commerce Carlos M. Gutierrez, Secretary

Technology Administration William Jeffrey, Acting Under Secretary for Technology 


\begin{abstract}
This paper describes the preliminary screening process that was conducted to identify potential candidate particles available from commercial sources to be used as National Institute of Standards and Technology (NIST) Standard Reference Material (SRM) for $30 \mathrm{~nm}$ spheres. Five different samples were obtained and measured using the NIST particle calibration facility. The sample with the narrowest size distribution and a preliminary peak particle diameter of $30.1 \mathrm{~nm}$ was identified as a potential candidate to become the NIST $30 \mathrm{~nm}$ SRM. A secondary candidate, with a fairly narrow distribution and a peak particle diameter of $24.2 \mathrm{~nm}$ was also identified. Further studies to address accuracy issues and ensure repeatability are recommended.
\end{abstract}

\title{
Introduction
}

An initial screening process to identify candidate particles that could be used as the NIST Standard Reference Material (SRM) $30 \mathrm{~nm}$ spheres was conducted. NIST currently has polystyrene latex (PSL) spheres available as SRMs as small as $60 \mathrm{~nm}$. The SRM particles are used primarily by the semiconductor industry to calibrate and test metrology instruments. The Semiconductor Industry Association has developed an International Technology Roadmap for Semiconductors (ITRS) specifying the need for smaller reference materials as semiconductor components and devices become smaller [1]. The ITRS discusses critical semiconductor components scaling to $32 \mathrm{~nm}$ or smaller by as early as 2008.

In response to the need for accurately sized particles, NIST has developed a calibration facility to accurately measure particles in the submicrometer range. The calibration facility uses electrostatic differential mobility analysis to measure the effective diameter of aerosolized particles. Compared to other methods, such as light scattering or electron microscopy, differential mobility analysis has lower uncertainty for measuring small particles and offers good statistics, because each test measures thousands of particles. Details regarding the calibration facility, including theory of operation and uncertainty assessments are included in the document by Donnelly and Mulholland [1]. Updates to the facility including the use of an electrospray aerosol generator and added uncertainty issues are discussed in Mulholland, et al. [2].

\section{Process}

NIST obtains commercially available submicron particles and accurately measures their size. To obtain the best possible sample to serve as the SRM for $30 \mathrm{~nm}$ particles numerous companies were contacted. Samples of monosize particles suspended in liquid, with a nominal diameter of $30 \mathrm{~nm} \pm 5 \mathrm{~nm}$ were acquired. One of the difficulties in obtaining the samples was that despite industry needs, many of the companies did not manufacture particles this small in diameter. In the end, five candidate samples were obtained. 
The samples were measured using the NIST particle calibration facility. A dilute liquid suspension containing the particles is placed into an electrospray aerosol generator where the particles are aerosolized. The particles then travel through an electrostatic classifier where they are separated by size using a controlled electric field. A monodisperse aerosol leaves the classifier and the particles are counted by a condensation particle counter. A computerized data acquisition system records the particle count and electrical field strength to determine the size and distribution of each sample.

Prior to screening the $30 \mathrm{~nm}$ samples, the system was calibrated using the SRM 1963, with a diameter of $100.7 \mathrm{~nm}$, whose measurement is traceable to the length standard. The system was checked to verify that the equipment was working properly and measurements were within the uncertainty range. During sample testing, calibration runs of the SRM 1963 were performed both before and after the sample measurements to correct for atmospheric conditions and any possible drift in the flows. Each candidate sample was tested twice and, the peak diameter was measured relative to the SRM 1963.

\section{Results}

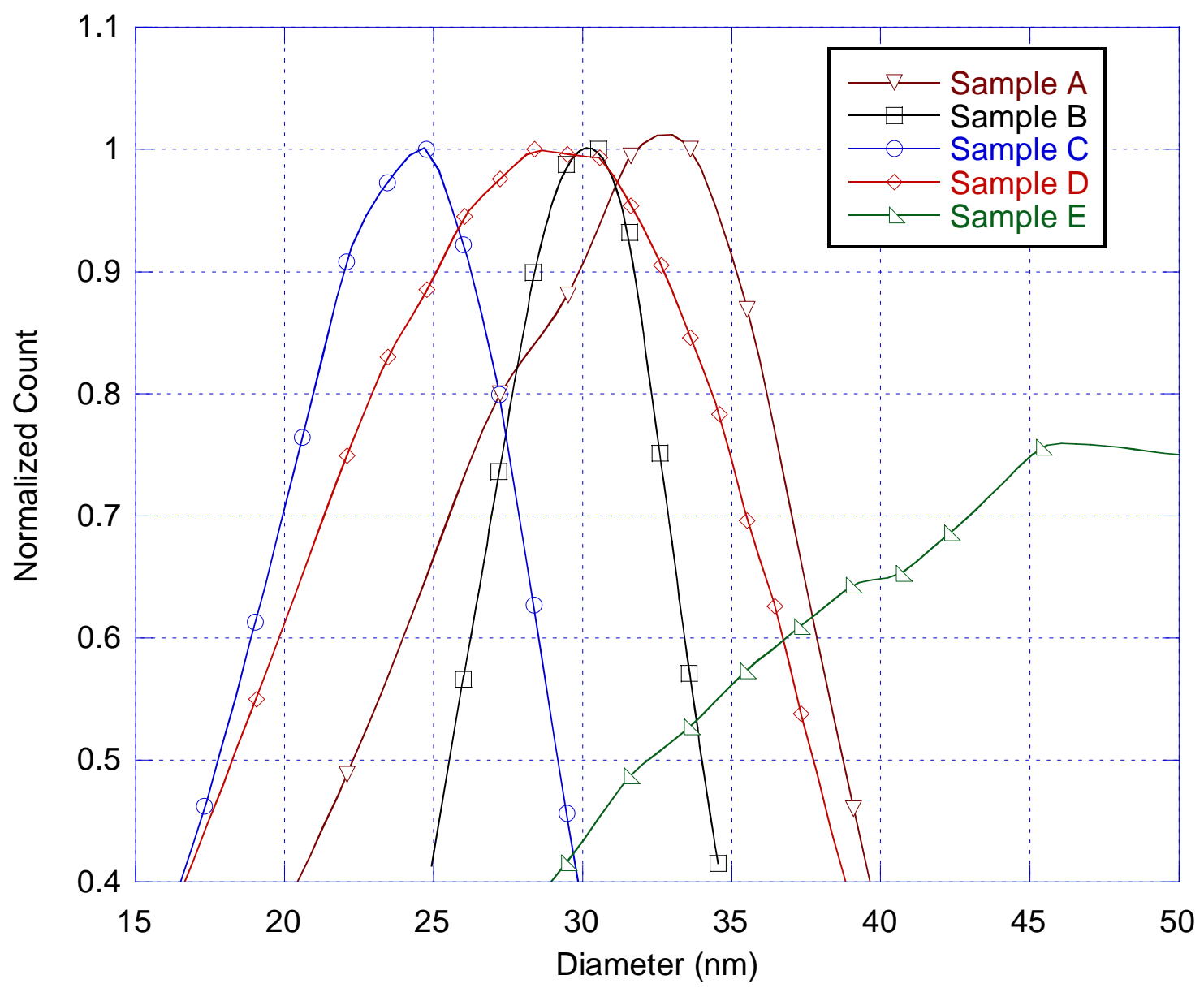

Figure 1. Particle size distribution as a function of the normalized count. 
The five candidate samples for the $30 \mathrm{~nm}$ SRM were measured using the NIST calibration facility, and the peak particle diameter was computed for each sample. The peak particle diameter represents the modal diameter, which is the peak in the number size distribution. The particle count for diameters between $15 \mathrm{~nm}$ and $50 \mathrm{~nm}$ was measured. Figure 1 shows the size distributions for the five candidate samples. Representative data points are included to distinguish the samples. Samples A, B, C, and $\mathrm{D}$ all have distinct peaks for the diameter range measured. The plot of Sample E shows that there was no discernable peak in the particle diameter over the size range of $15 \mathrm{~nm}$ to $50 \mathrm{~nm}$, as the number concentration continually increased over the size range examined. The preliminary, uncorrected mean peak diameters and the estimated uncertainty in the diameter measurements are listed in Table 1. For Sample E, a peak was not identified in this range.

Table 1. Preliminary mean peak diameter and estimated uncertainty.

\begin{tabular}{|c|c|c|}
\hline Particle Sample & Preliminary Peak Diameter (nm) & Estimated Uncertainty (nm) \\
\hline A & 32.1 & 0.9 \\
\hline B & 30.1 & 0.8 \\
\hline C & 24.2 & 1.0 \\
\hline D & 29.2 & 1.4 \\
\hline E & undetermined & N/A \\
\hline
\end{tabular}

When selecting a sample to be used as an SRM, it is desirable for the peak of the distribution to be close to the target diameter, in this case $30 \mathrm{~nm}$. Peak size, however, is not the only criteria for a good sample. The size distribution should be fairly symmetric, with no shoulders or ledges on the sides of the peak. A narrow distribution culminating with a steep peak is most desirable. A quantitative way to define the narrowness of the sample peaks is to compare the widths of the distributions as a function of the normalized particle count. The widths of the distribution were found using the plots of the particle size distributions. The width of the distribution was measured for each sample at the normalized particle count of 0.8 and 0.6. Figure 2 shows an example of the distribution width measurements conducted for Sample B. The measured widths at 0.8 and 0.6 for all of the samples, excluding sample E, are listed in Table 2 . Of the nominally $30 \mathrm{~nm}$ samples tested, sample B had the narrowest distribution, measuring $4.7 \mathrm{~nm}$ at 0.8 and $7.2 \mathrm{~nm}$ at 0.6. For reference, the width of the distribution for the $100 \mathrm{~nm}$ SRM (SRM 1963) is $3.0 \mathrm{~nm}$ at 0.8 and $4.5 \mathrm{~nm}$ at 0.6 .

Table 2. Width of the distribution at normalized particle count.

\begin{tabular}{|c|c|c|}
\hline Sample & Width (nm) at 0.8 & Width (nm) at 0.6 \\
\hline A & 9.0 & 13.9 \\
\hline B & 4.7 & 7.2 \\
\hline C & 6.5 & 9.4 \\
\hline D & 11.5 & 16.7 \\
\hline E & N/A & N/A \\
\hline
\end{tabular}




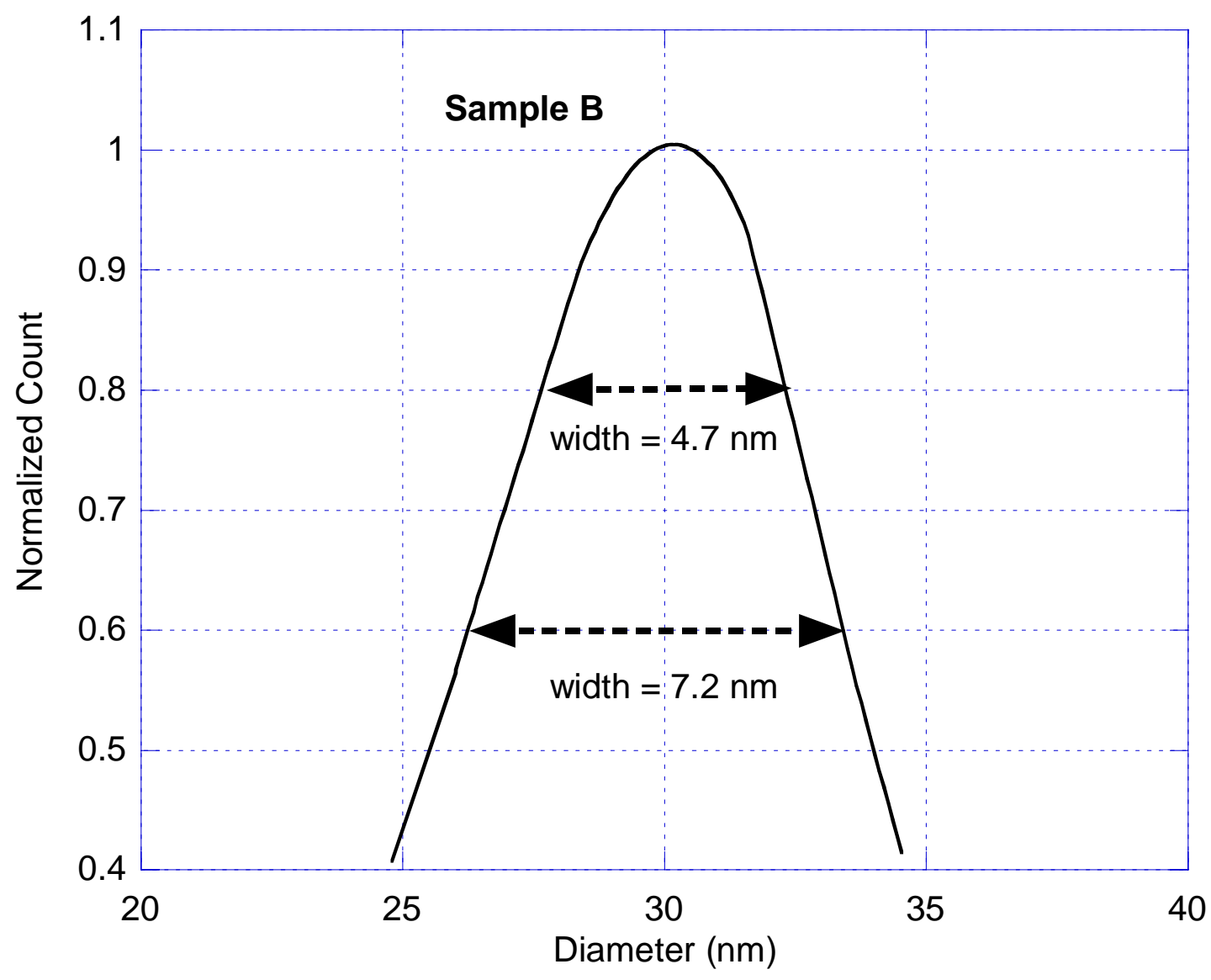

Figure 2. Graph of the width of the distribution for Sample B.

Based on the criteria for selecting a sample to become the nominal $30 \mathrm{~nm}$ SRM, Sample B is the best candidate, because it has the narrowest and most symmetrical size distribution. The preliminary peak diameter of $30.1 \mathrm{~nm}$ is also very close to the target diameter of $30 \mathrm{~nm}$. The second choice would be Sample C. Its size distribution is slightly wider than Sample B, but is fairly symmetrical. Also, the peak diameter of Sample C is small, measuring $24.2 \mathrm{~nm}$, so it would be a nominally $25 \mathrm{~nm}$ sample.

For screening purposes, two measurement runs were performed for each sample in order to obtain preliminary measurements of the peak particle size and the distribution. When determining the certified measurements for the SRM certificate, a series of at least 10 measurements are performed to obtain accurate statistics and uncertainty. Additionally, the results given here for the peak diameters are the preliminary, uncorrected measurements used to screen the candidates. It is possible to have a small drift of the electrospray generator flow during the sample run which can slightly skew the measurement. Error due to this drift can be minimized and corrected by taking drift measurements during the sample test, but it was not done here because the error is small (approximately $0.07 \mathrm{~nm}$ ) and this was preliminary screening. Another source of error 
that can effect the measurements is the transfer function of the electrostatic classifier. The transfer function is the probability that a particle entering the classifier will exit through the passage at the end of the differential mobility analyzer [3]. Due to the operating principle of the system as the particle size increases, slightly more of the larger particles are counted, shifting the peak count to a larger number. Because the particles are nominally the same size, the transfer function correction would be nominally the same for all of the samples and would not be necessary during the preliminary screening.

\section{Conclusions}

Five candidate samples were obtained and tested, and four of the five had peak diameters within $6 \mathrm{~nm}$ of the target diameter of $30 \mathrm{~nm}$. The size distributions of these four samples were fairly symmetrical, though not as narrow as the $100 \mathrm{~nm} \mathrm{SRM.} \mathrm{Of} \mathrm{the} \mathrm{samples}$ tested, sample $B$ is the best choice to become the $30 \mathrm{~nm}$ particle standard. It has the narrowest size distribution with a steep symmetrical peak. The preliminary, uncorrected peak particle diameter for sample B is $30.1 \mathrm{~nm}$. The estimated uncertainty is $\pm 0.8 \mathrm{~nm}$.

\section{Recommendations}

The interest for smaller SRM particles is present in the semiconductor industry. In order to have a well defined SRM in the $30 \mathrm{~nm}$ range, continuing work is needed. Multiple samples of the prime candidate should be obtained and measured for repeatability. Numerous measurements should be performed on varying days, and accounting for drift within the measurement should be taken. The issue of the transfer function would need to be investigated and the uncertainty computed for the $30 \mathrm{~nm}$ size. Also, the possible deposition of residue on the particles would need to be considered. Additional measurements using other methods, such as light scattering would be helpful in supplementing the work.

\section{References}

[1] Semiconductor Industry Association. International Technology Roadmap for Semiconductors (ITRS): 2005 Edition, available at http://public.itrs.net.

[2] Donnelly, M. K., and Mulholland, G. W. "Particle Size Measurements for Spheres With Diameters of 50 nm to 400 nm,” NISTIR 6935; August 2003.

[3] Mulholland, G. W., Donnelly, M. K., Hagwood, C., Kukuck, S. R., and Hackley, V. A., "Measurement of $100 \mathrm{~nm}$ and $60 \mathrm{~nm}$ Particle Standards by Differential Mobility Analysis," Journal of Research of the National Institute of Standards and Technology, Vol. 111, No. 4, 257-312, July-August 2006.

[4] Donnelly, M. K., Mulholland, G. W. and Winchester, M.R. "NIST Calibration Facility for Sizing Spheres Sustpended in Liquids," AIP Conference Proceedings 683, Characterization and Metrology for ULSI Technology: 2003 International Conference, Austin, Texas, 313-317, March 2003. 